UNIVERSIDADE ESTADUAL DE FEIRA DE SANTANA

Autorizada pelo Decreto Federal $n^{\circ} 77.496$ de 27/04/76

Recredenciamento pelo Decreto $n^{\circ} 17.228$ de $25 / 11 / 2016$

PPPG

PRÓ-REITORIA DE PESQUISA E PÓS-GRADUAČ̃̃

COORDENAÇÃO DE INICIAÇÃO CIENTÍFICA

XXIV SEMINÁRIO DE INICIAÇÃO CIENTÍFICA DA UEFS

SEMANA NACIONAL DE CIÊNCIA E TECNOLOGIA - 2020

\title{
DEFICIÊNCIA DE TESTOSTERONA E OBESIDADE: PREVALENCIA NA REGIÃO METROPOLITANA DE FEIRA DE SANTANA-BA
}

\author{
$\underline{\text { Ana Carolina Silva Assuncão }}{ }^{1}$; Monique Tonani Novaes ${ }^{2}$; José de Bessa Júnior $^{3}$ e \\ Carla Jamile Jabar Menezes ${ }^{4}$ \\ 1. Bolsista PROBIC/UEFS, Graduanda em Medicina, Universidade Estadual de Feira de Santana, e-mail: \\ anacarolina_assuncao@homail.com \\ 2. Programa de Pós-Graduação em Saúde Coletiva, Universidade Estadual de Feira de Santana, e-mail: \\ moniquetonani@yahoo.com.br \\ 3. Orientador do grupo UROS e subgrupos populacionais, Departamento de saúde, Universidade Estadual de Feira \\ de Santana, e-mail: josedebessa@gmail.com \\ 4. Orientadora, Departamento de saúde, Universidade Estadual de Feira de Santana, e-mail: carlamenez@ gmail.com
}

PALAVRAS-CHAVE: testosterona; obesidade; deficiência.

\section{INTRODUÇÃO}

A obesidade é um agravo de caráter multifatorial proveniente do balanço energético positivo que favorece o acúmulo de gordura, associado a riscos para a saúde devido à sua relação com complicações metabólicas, como aumento da pressão arterial, aumento dos níveis de colesterol, triglicerídeos sanguíneos e resistência à insulina (WHO, 2000). De acordo com o National Cholesterol Education Program - Adult Treatment Panel III, o ponto de corte para diagnóstico da obesidade a partir da avaliação da circunferência abdominal (gordura visceral) em homens deve ser $102 \mathrm{~cm}$ (ABESO, 2016).

A deficiência de testosterona em homens ocorre quando há presença de baixos níveis séricos de testosterona e espermatozoides, acompanhados de sinais e sintomas clínicos (BHASIN, et al., 2010). A deficiência primária (testicular) e a deficiência secundária (cascata pituitária-hipotalâmica) são acompanhadas de sintomas (BHASIN, 2008). A deficiência de testosterona é definida como níveis séricos de testosterona total abaixo de $300 \mathrm{ng} / \mathrm{dL}$ e níveis de testosterona livre abaixo de 6,5 ng/dL (MARTITS, 2004).

A testosterona é um hormônio que desempenha papel essencial no metabolismo de carboidratos, gorduras e proteínas. Dessa maneira, influencia na composição da gordura corporal e na massa muscular no homem. A deficiência de testosterona está associada a um aumento da massa gorda (principalmente obesidade central), redução da sensibilidade à insulina, tolerância à glicose diminuída, triglicerídeos e colesterol elevados e baixo HDL-colesterol (KELLY; JONES, 2013).

Os níveis baixos de testosterona estão associados à obesidade, mas a direção e a causalidade dessa relação ainda não são claras. É importante destacar a obesidade como um fator de risco para baixos níveis séricos de $\mathrm{T}$ em homens, com implicações em possíveis estratégias relevantes de promoção da saúde (ERIKSSON et al, 2017). 
A obesidade está associada a uma infinidade de doenças, como: doenças cardiovasculares, apneia do sono, osteoartrite, aumento do risco de certos cânceres e, nos homens, redução dos níveis de testosterona. Dados sugerem que o único e mais poderoso preditor de deficiência de testosterona é a obesidade (FUI et al, 2014).

O envelhecimento por si só é raramente associado a reduções acentuadas nos níveis de testosterona. A disfunção testicular relacionada à idade é compensada por aumento associado a secreção hipofisária de hormônio luteinizante (LH). No entanto, a obesidade enfraquece esse aumento de LH e então leva à supressão do eixo hipotálamohipófise, o que independe da idade, e que não pode ser compensada por mecanismos fisiológicos (FUI et al, 2014).

\section{METODOLOGIA}

Trata-se de estudo observacional retrospectivo a partir de um banco de dados sob a guarda do nosso grupo de pesquisa. $\mathrm{O}$ banco de dados foi obtido a partir de pacientes do sexo masculino, onde constam informações sobre: idade, presença de hipertensão, circunferência abdominal, níveis de glicose e do perfil lipídico, valor de antígeno prostático especifico, valor de ureia, creatinina, vitamina $\mathrm{D}$, além dos valores dos níveis séricos de testosterona. Nas comparações, a deficiência de testosterona foi definida como testosterona sérica inferior a 300ng/dL, conforme preconizado na literatura. Obesidade foi definida como circunferência abdominal (CA) maior ou igual a $102 \mathrm{~cm}$. As variáveis quantitativas, contínuas ou ordinais, foram descritas por suas medidas de tendência central (médias ou medianas) e pelas respectivas medidas de dispersão (desvio-padrão, variação interquartil ou valores mínimo e máximo), enquanto as nominais ou qualitativas por seus valores absolutos, percentagens ou proporções. Para comparação das diferenças das variáveis contínuas, utilizamos o teste t de Student ou o teste de Mann-Whitney. Teste de D'Agostino-Pearson foi empregado na avaliação do padrão de distribuição das variáveis. Na comparação dos dados categóricos, utilizamos os testes de Fisher ou do quiquadrado e suas variantes. A associação entre os parâmetros estudados foi expressa pela razão de prevalência ou Odds Ratio. Intervalos de confiança de $95 \%$ foram empregados como medidas de precisão dos resultados. Valores de $p$ menores que $0,05(p<0,05)$ foram considerados significativos. Nas análises foi utilizado o programa estatístico computacional GraphPad Prism, versão 7.0.3, GraphPad Software, San Diego-CA, USA.

\section{RESULTADOS E DISCUSSÃO}

O estudo incluiu 2796 homens, com idade de 57 [50-65] anos. Obesidade foi observada em 34\% (960) e a deficiência de testosterona em 20,6\% (577). A distribuição desses dados está demonstrada na figura a seguir:

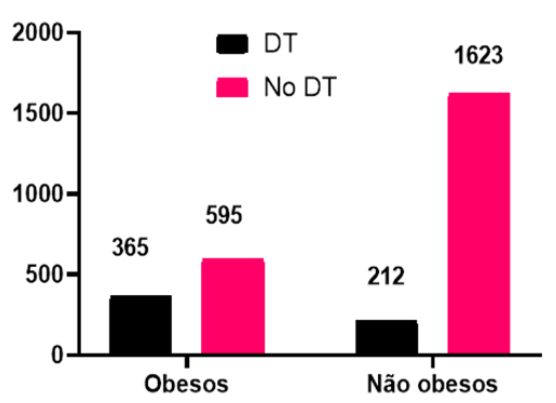

Figura 1: associação obesidade e deficiência de testosterona em indivíduos do sexo masculino em Feira de Santana-BA. 
As medianas da testosterona e circunferência abdominal foram, respectivamente, 413 [315-532] ng/dl e 98 [92-105] cm. A prevalência de deficiência de testosterona foi significativamente maior nos obesos que nos não obesos, respectivamente $38 \%$ (365/960) x 11,5\% (212/1835). OR=4,7[3,9-5,7] IC95\% ( $<<0,0001)$. A figura 2 demonstra os dados:

Circinferência Abdominal $(\mathrm{cm})$

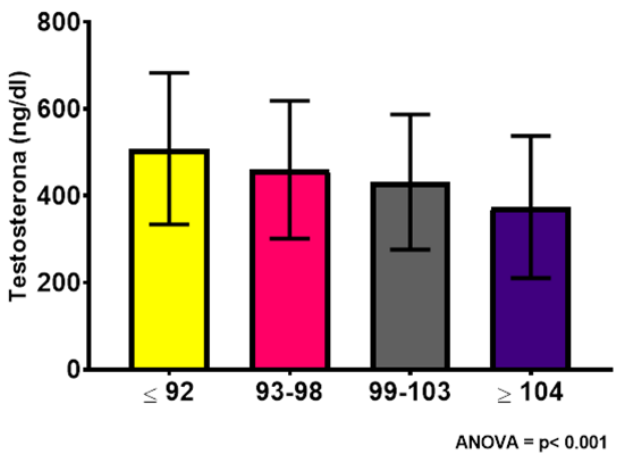

Figura 2: variação da testosterona conforme intervalo interquartil em indivíduos do sexo masculino.

A análise da curva ROC revelou que a circunferência abdominal tem uma acurácia de $72 \%$ no diagnóstico de deficiência de testosterona. Circunferência abdominal $\geq 102 \mathrm{~cm}$ foi o melhor valor de corte com uma sensibilidade de $61 \%$ e especificidade de $75 \%$, o que em nosso cenário possibilita um valor preditivo negativo de $89 \%$. A figura 3 demonstra os dados:

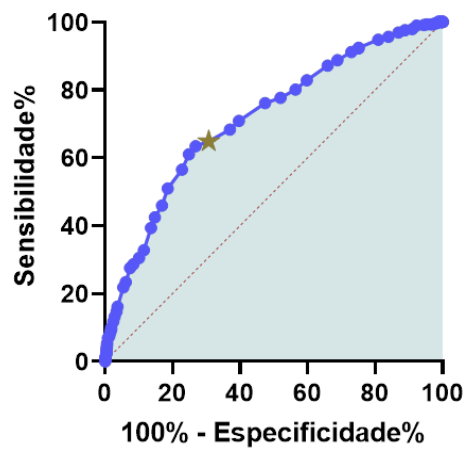

Figura 3: análise da curva ROC em indivíduos do sexo masculino.

Tajar et al (2010) demonstrou em uma coorte de 3219 homens com idades entre 40-79 anos do European Male Aging Study (EMAS), que 35,1\% dos indivíduos tem circunferência abdominal $\geq 102 \mathrm{~cm}$. A obesidade foi associada a um risco relativo de hipogonadismo secundário (definido como testosterona total $<10,5 \mathrm{nmol}$ ) de 8,7 vezes $(p<0,001)$ em relação ao peso normal. O presente estudo mostrou que o hipogonadismo secundário está associado à obesidade independentemente da idade.

Rohrmann et al (2011) mostrou em um estudo com 1.265 homens do Terceiro National Health and Nutrition Examination Survey (NHANES III) que 24,5\% tinham circunferência abdominal $\geq 102 \mathrm{~cm}$ e que as concentrações de testosterona total diminuíram com o aumento da gordura corporal.

Eriksson et al (2017) sugeriu a partir da análise de randomização mendeliana bidirecional em 5 coortes prospectivas com 7.446 homens, que existe um efeito causal do IMC na testosterona sérica em homens. Intervenções para reduzir o IMC aumenta a testosterona sérica nos homens. 
Carrageta et al (2019) mostrou que há um ciclo vicioso entre hipogonadismo e aumento da adiposidade, ou seja, a obesidade pode ser uma causa para o hipogonadismo masculino, assim como o hipogonadismo pode ser causa para a obesidade. Na direção oposta, a perda de peso sustentada pode atingir a melhora da função gonadal masculina.

\section{CONSIDERAÇÕES FINAIS}

Observamos em nossa amostra significativa prevalência de obesidade e DT, e uma forte associação entre as duas condições. $\mathrm{O}$ aparente efeito protetor da idade nos valores séricos e na homeostase da $\mathrm{T}$, conceito muito arraigado e difundido no passado, provavelmente foi contraposto pela obesidade epidêmica nos dias atuais. A força da associação e o alto valor preditivo da CA sugere que a dosagem da T sérica esteja indicada nos obesos.

\section{REFERÊNCIAS}

ASSOCIAÇÃO BRASILEIRA PARA O ESTUDO DA OBESIDADE E DA SÍNDROME METABÓLICA. Diretrizes brasileiras de obesidade. 4. ed. São Paulo: ABESO, 2016.

BHASIN S. Testicular disorders. In: Larsen PR, Kronenberg HM, Melmed S, Polanski KS, eds. Williams' textbookofendocrinology. 11th ed. Philadelphia: Elsevier. 2008.

BHASIN S.; CUNNINGHAM G.R.; HAYES F.J.; MATSUMOTO A.M.; SNYDER P.J.; SWERDLOFF R.S.; MONTORI V.M.; TASK FORCE, ENDOCRINE SOCIETY. Testosterone therapy in men with androgen deficiency syndromes: na Endocrine Society clinical practice guideline. J ClinEndocrinolMetab. 2010, v.95, n.6, p.2536-2359.

CARRAGETA, D.F.; OLIVEIRA, P.F.; ALVES, M.G.; MONTEIRO, M.P. Obesity and male hypogonadism: Tales of a vicious cycle. Obesity Reviews. 2019; 20: 1148-1158.

ERIKSSON, J. et al. Causal relation ship between obesity and serum testosterone status in men: A bi-directional mendelian randomization analysis. PloS one, v. 12, n. 4, p. e0176277, 2017.

FUI, M.N.T.; et al. Lowered testosterone in male obesity: mechanisms, morbidity and management. Asian journal of andrology, vol. 16, n. 2 p. 223-231, 2014.

KELLY, D. M.; JONES, T. H. Testosterone: a metabolic hormone in health and disease. J Endocrinol. 2013 Apr 29;217(3):R25-45.

KELLY, D. M.; JONES, T. H. Testosterone and obesity. Obes Rev. 2015 Jul;16(7):581-606.

MARTITS, A.M.; COSTA E.M.F. Diretrizes em Foco. Medicina Baseada em Evidência. Hipogonadismo masculino tardio ou andropausa. Rev Assoc Med Bras. 2004, v.50, n.4, p. 349362.

ROHRMANN, S.; SHIELS, M.S.; LOPEZ, D.S. et al. Body fatness and sex steroid hormone concentrations in US men: results from NHANES III. Cancer Causes Control. 2011;22(8):11411151.

TAJAR, A.; FORTI, G.; O'NEILL, T.W. et al. Characteristics of secondary, primary, and compensated hypogonadism in aging men: evidence from the European Male Ageing Study. J Clin Endocrinol Metab. 2010;95(4):1810-1818.

WORLD HEALTH ORGANIZATION. Obesity: preventing and managing the global epidemic. Report of a WHO consultation on obesity. Geneva, Switzerland: WHO, 2000. (WHO Technical Report Series, no. 894). 